\title{
Manini diseñador en la Quinta da Regaleira: el lenguaje clásico de una obra "neomanuelina" (1898-1912)
}

\section{Manini designer at the Quinta da Regaleira: the classi- cal language of a "neomanueline" work (1898-1912)}

\author{
IVÁn Moure Pazos \\ Istituto di Studi Superiori - Università di Bologna; \\ CIAUD -Faculdade de Arquitectura- Universidade de Lisboa; \\ Departamento de Historia da Arte - Universidade de Santiago de Compostela \\ ivan.menes@yahoo.es
}

Recibido: $14 / 03 / 2016$

Aceptado: 21/09/2016

\section{Resumen}

Entre 1898 y 1912, Luigi Manini proyecta en la sierra de Sintra la obra más representativa de su prolífica carrera artística: la Quinta da Regaleira. Se trata de una "obra de arte total" llamada a erigirse como el edificio más representativo del neomanuelino finisecular portugués. Sin embargo, y como veremos a continuación, esta etiqueta estilística -ampliamente socializada y comúnmente oficializada- pudiera ser revisada, a tenor de las múltiples y profusas "contaminaciones figurativas" de corte clásico que Manini introduce en su gramática decorativa. Si bien es cierto que las grandes obras manuelinas del XVI -de predominancia claramente "medievalizante"- estuvieron siempre salpimentadas de pequeñas concesiones al mundo italianizante y romano, en el caso de la Regaleira se da una desproporción estilística a favor de este último que equilibra ambos pensamientos artísticos. Por lo tanto, el presente artículo sostiene la tesis de que en dicho pedernal conviven dos lenguajes antagónicos muy bien medidos y proporcionados: lo clásico y lo medieval. Éstos, a modo de "bisagras estilísti- 
cas", se entrelazan, facilitando la convivencia de ambas tradiciones artísticas. El fin último de este ejercicio de eclecticismo estriba en la consecución armónica de un conjunto fuertemente integrador, bien conjugado y proporcionado. A través de la creación de nuevas hibridaciones figurativas, Manini logra el "milagro" conciliador entre sendos mundos encontrados. Por lo tanto, el artículo propone una relectura de la Regaleira en base a un nuevo enfoque, centrado en la continua reutilización y reinvención de la retórica clásica que, Manini, a través de sus diseños ornamentales, introduce en la Regaleira, neutralizando así aquellos excesos medievales propios del manuelino y dotando al conjunto de una cierta proporcionalidad renacentista. Todo ello obligará a una redefinición estilística del programa decorativo de la Regaleira, donde, quizás, tengamos que hablar más de un "neomanuelino clasicista" que de un "neomanuelino ortodoxo" de clara predominancia medieval.

\title{
Palabras clave
}

Luigi Manini, Quinta da Regaleira, neomanuelino, neorrenacimiento.

\begin{abstract}
Between 1898 and 1912, Luigi Manini projected in the mountains of Sintra the most representative work of his prolific career: the Quinta da Regaleira. The building was a "total work of art", which destiny will be to stand as the most representative work of finisecular neomanueline, portuguese architecture. However, as will be discussed below, this stylistic label -widely socialized and commonly officialized- may be revised, according to the many and profuse stylistic elements of a classic background that have contaminated Manini's decorative grammar. Even if the main manueline works of XVI century - clearly dominated by a medieval like style - have always been seasoned with small italian and classic references, when we talk about Quinta da Regaleira, it is made evident that there is a stylistic disproportion in favor of the second one, that equilibrates both of them artistic styles. Hence, this article tries to illustrate the presence of two very well measured and proportionate antagonistic stylistic languages: classical and medieval. Both functions as "stylistic hinges", intertwined, facilitating the coexistence of both artistic traditions. The ultimate goal of such displaying of eclecticism is the harmonious achieving of a highly integrated set, both nicely conjugated and proportionate. Through the creation of new figurative hybridizations, Manini achieved the conciliator "miracle" between two opposite worlds. Therefore, this paper proposes a rereading of the stylistic analysis of the named work, on the basis of a new approach, focused on the continuous reuse and reinvention of the classical rhetoric, that Manini introduced in the Quinta da Regaleira through his ornamental scheme, which final result is the neutralization of medieval excesses which characterize the manueline, and providing the set of a certain renaissance proportionality.
\end{abstract}


All this will force a stylistic redefinition of the decorative program of Regaleira, in which, perhaps, we should better talk about a "classic neo manueline" than an "orthodox manueline" with a clear medieval predominance.

\section{Keywords}

Luigi Manini, Quinta da Regaleira, neomanueline, neorenaissence.

Referencia normalizada: MOURE PAZOS, IVÁN (2016): “Manini diseñador en la Quinta da Regaleira: el lenguaje clásico de una obra "neomanuelina" (1898-1912)". Arte y Ciudad. Revista de Investigación, no 10 (octubre), págs. 7-29. Madrid. Grupo de Investigación Arte, Arquitectura y Comunicación en la Ciudad Contemporánea, Universidad Complutense de Madrid.

Sumario: 1.- Acercamiento a una biografía intelectual. 2.- Manini diseñador y ornamentista en la Quinta da Regaleira. 3.- Diseño exterior: predominancia neomedieval y contaminaciones clásicas (e hibridación de ambos léxicos). 4.- Diseño interior: predominancia clásica y contaminaciones neomedievales. 5.- Conclusiones. 6.- Bibliografía.

\section{Acercamiento a una biografía intelectual ${ }^{1}$.}

Con motivo del octogésimo aniversario de la muerte de Luigi Pietro Manini (1848-1936) -en adelante Manini- consideramos justo dedicar un merecido homenaje al afamado pintor, diseñador, ornamentista, fotógrafo, paisajista, escenógrafo y arquitecto lombardo.

La vida de Manini pudiera parangonarse a la de una gran epopeya épica; una obra de arte en sí misma. Su biografía ha sido tratada ampliamente por reputados estudiosos portugueses e italianos, los cuales han puesto en solfa sus principales conquistas artísticas (D'Acie, 1936: 14; Pereira; Luckhurst, 2006a: 11-19; Piccarolo, 2006: 389-391; Ricci, 2007: 14-15).

\footnotetext{
${ }_{1}$ El presente artículo forma parte del proyecto internacional Laboratorio di Ricerca sulle Città (Istituto di Studi Superiori. Università di Bologna). Asimismo, también del proyecto europeo Performigrations: people are the territory (Dipartimento di Lingue, Letterature e Culture Moderne. Università di Bologna). Análogamente, dicho artículo ha sido posible gracias a la financiación postdoctoral del Plan Galego de Investigación, Innovación e Crecemento 2011-2015 promovido por la Xunta de Galicia. Gran parte de la bibliografía de este artículo se encuentra editada únicamente en italiano y portugués. Todos los textos citados han sido traducidos por el autor al castellano.
} 
Manini nace en Crema, Lombardía, en el seno de una familia de escasos recursos económicos. Este hecho condicionará su desarrollo educativo, restringiendo su tiempo de formación académica a un único año lectivo. Concretamente, sabemos por Gaia Piccarolo que Manini asistió como aplicado discente a la cátedra de ornato en la Accademia di Belle Arti di Brera de Milano durante el curso 1861-62 (2006: 389). Su punto de partida fue, por lo tanto, diferente a la de sus coetáneos colegas de oficio, como Angelo Bacchetta, Giovanni Vela u otros artistas afines formados en torno al afamado círculo de Brera. Sin embargo, Manini hizo ventaja de la desventaja, o como reza el dicho español "hizo de la necesidad virtud", convirtiéndose, andando el tiempo, en el artista más estimado y apreciado de su generación.

Manini fue el último hombre del renacimiento italiano, un artista irrepetible e inimitable al que, quizás, no se le haya prestado demasiada atención en España². Suele emplearse con bastante frecuencia la expresión de "hombre del renacimiento", para designar a aquellos artistas que, movidos por una insaciable curiosidad universal, abordan con éxito diversas disciplinas del saber humanístico. Pero con demasiada asiduidad, olvidamos que el mundo racionalista y académico imperante en el siglo XVIII, supuso el fin definitivo de un modelo de formación gremial, artesanal y renacentista - de tintes tan medievalizantes como románticos-, que basó su sistema de aprendizaje en la estrecha relación maestro-discípulo. Dicho sistema vertebró, como sabemos, uno de los capítulos más interesantes de la historia del arte occidental: el Renacimiento italiano. No está de más recordar que, muchos de los artistas contemporáneos adscritos a esta designación humanista, forman parte firmante de los nuevos postulados de aprendizaje modernos y los saberes hiperespecializados. No fue el caso de Manini que, en 1857, y con tan sólo 9 años de edad, ingresa como aprendiz en el atelier del célebre pintor Giovanni Zaffeva (Pereira; Luckhurst, 2006a: 13). Cuatro siglos antes, Michelangelo Buonarroti, hacía lo propio en el taller de los Ghirlandaio. Se trataba de la misma tradición formativa trasladada a la realidad contemporánea. Por entonces, éste continuaba siendo un método de aprendizaje alternativo al de las academias oficiales, sobre todo entre la gente de condición más humilde que debía procurarse un jornal. El sistema, a juzgar por los resultados obtenidos -a la historia del arte

\footnotetext{
${ }^{2}$ La única aportación científica aparecida sobre Manini en lengua castellana en Moure Pazos (2014).
} 
nos remitimos-, resultaba más que valido, favorecido, quizás, por el trato particularizado del maestro al joven artista que ingresaba en el atelier.

Manini fue uno de los hijos pródigos que, a lo largo de la historia, formaron parte de este sistema formativo, proporcionándole una base más que sólida para el desempeño de sus posteriores habilidades artísticas. Así, a lo largo de su período italiano (1848-1878), trabaja en infinidad de palacios, villas e iglesias tanto en Crema como en sus alrededores, granjeándose una estimable reputación como artista decorativo y eficiente restaurador de frescos. En 1873, haciendo gala de una versatilidad artística difícilmente parangonable, ingresa como escenógrafo-aprendiz en el Teatro alla Scala de Milano, sustituyendo, cinco años más tarde, a su regidor titular: Carlo Ferrario. En esta etapa se gesta y desarrolla la seña identitaria que caracteriza sus grandes aportaciones del período portugués: el continuo diálogo artístico e intermedial entre arquitectura, escenografía y paisaje (D'Amia, 2007: 59; Alpini, 2007: 117-118):

La búsqueda de los mejores ángulos, de las perspectivas más favorables, le llevó a caminar por la montaña, aspecto que se evidencia en las fotos que quitó de Sintra. Dichas fotos, tienen el poder de revelar la imaginación de Manini. Detrás de la máquina fotográfica, se coloca en la posición de observador, de localizador, buscando hacer suyo el espíritu del lugar [...] reflejado en sus intervenciones paisajísticas de Sintra [...], existiendo un perfecto paralelismo entre el constructor de paisajes y el inventor de la escena. Este universo (pintura y arquitectura) se entrelazan en su proceso creativo, patentizando el hecho de que la observación de la naturaleza y la narrativa lírica se transforman en los principales soportes de la conceptualización del artista [...]. Esta afirmación se hace evidente en la colina de Sintra. Los edificios de Manini fueron concebidos como elementos constituyentes y generadores de nuevos paisajes. Subrayando el aura del Palacio da Pena, del Castelo dos Mouros, del Palacio Real, sus proyectos recrean el paisaje sintrense como si fuese irreal [...] (Luckhurst; Pereira, 2007: 89-90).

Y sobre ello vuelve también Iván Moure en su artículo de reciente aparición sobre las Villas de Luigi Manini en Sintra:

Gaia Piccarolo, en su obra Luigi Manini (1848-1936). L'opera architettonica di uno scenografo italiano in Portogallo (2005), ha sido la primera en establecer un vínculo claro entre el pasado escenográfico de Manini y su posterior desarrollo como arquitecto, llegando a considerar la Villa Mayer como proyecto de "alta inspiración escenográfica" y ejemplo claro de esta continua dialogicidad. Dicho diálogo va más allá de lo meramente paisajero. No se trata solo de una influencia implícita 
(la búsqueda de un genius loci supeditado a la orografía del terreno y la optimización de perspectivas visuales -anteriormente abordadas-), sino que, ésta, se explicita en los propios proyectos arquitectónicos del artista. De tal modo que, muchas de las obras delineadas, ya estaban contenidas en precedentes proyectos operísticos y teatrales, al modo de plantillas constructivas (Moure, 2014: 110).

Tal es el caso, también, de su afamada Villa Sassetti (1890) en Sintra, contenida ya en algunas pinturas precedentes como Veduta fantástica di città con castello (1862), entre muchas otras. Por lo tanto, podremos establecer una clara linealidad artística entre las primeras obras decorativas, pictóricas y escenográficas de su etapa italiana y las grandes intervenciones arquitectónicopaisajistas de su posterior período portugués.

En 1879, Manini deja Italia rumbo a Portugal para emplearse como afamado escenógrafo en el Real Teatro San Carlos de Lisboa. Allí trabajará sin descanso durante más de 30 años, convirtiéndose en el arquitecto y paisajista predilecto de la alta burguesía lisboeta (Pereira; Luckhurst, 2006b: 44). Y es que, pese a su carácter fuertemente esquivo, tímido y misántropo, logrará la simpatía de la selecta sociedad de la época, entre la que se contaban grandes personalidades de la cultura, la realeza y la aristocracia del momento.

Debe reseñarse que Manini carecía de formación como arquitecto, siendo un auténtico autodidacta en esta disciplina (Piccarolo, 2005: 272-276). Ésto no le impidió el abordaje de empresas complejísimas, estudiando por cuenta propia diferentes aspectos funcionales, como el cálculo de estructuras, la resistencia de materiales o la aplicación de diversos sistemas constructivos. Desafiando a las más estrictas leyes de la gravedad, erigió excelentes pedernales en terrenos escarpados y difíciles orografías de montaña como las vistas en la sierra de Sintra o el bosque de Buçaco (Henriques da Silva, 2004: 44-49; Anacleto, 2004: 64-73; Pereira; Luckhurst, 2004: 50-63, Valdeón, 2008: 14-15; Moure, 2014: 101-116).

Actualmente, sus obras forman parte firmante y fundamental de cualquier catálogo razonado de arquitectura contemporánea. Casa del Giardiniere-Biester (1890-1891), Villa Sassetti (1890), o el Palácio de Jorge O’Neill (1897) son, hoy, clásicos indispensables de la historia del arte europeo (Pereira; Luckhurst, 2004b: 253-280; Pereira; Luckhurst, 2006c: 77-89; Piccarolo, 2007: 99-107; Pereira; Luckhurst, 2008: 200-210; Pereira; Luckhurst, 2011: 92-105; Pereira, 2012: 
147-159). Pero, sobre todo, la fama de Manini ha trascendido a nivel internacional debido a la creación de dos obras monumentales, llamadas a ser los buques insignia del neomanuelino luso: el gran Palaço de Buçaco (1888-1907) en Coimbra y la suntuosa Quinta da Regaleira (1898-1912) en Sintra (Pereira; Luckhurst, 2004: 50-63; Carita, 2006a: 90-98). Se trata de dos obras colosales de excelente factura técnica que debemos encuadrar dentro de la ambiciosa designación wagneriana de "obra de arte total". [Figs. 1-2].

Caso rarísimo en el panorama de la arquitectura portuguesa de la época, el proyecto de la Quinta da Regaleira emerge como ejemplo de una obra de arte total, convocando la participación de una multiplicidad de prácticas artísticas asumidas aquí por un único protagonista (Carita 2006a: 122).

\section{Manini diseñador y ornamentista en la Quinta da Regaleira.}

Manini fue un excelente diseñador, decorador y ornamentista. Debemos recordar, nuevamente, que su único año de formación académica se desarrolló en la cátedra de ornamento de esta misma disciplina, destacando como alumno aventajado de su promoción (Carita, 2006a: 106). Sin embargo, resulta evidente que su fama de paisajista y arquitecto ha eclipsado con creces sus prolíficas aportaciones a los campos del diseño y el ornamento. Existe consenso en considerar a la Quinta da Regaleira como su obra más ambiciosa y completa; un enorme pedernal que configuró y proyectó en su práctica totalidad, conjuntamente a su mecenas Augusto Carvalho Monteiro. Se trata de un lúgubre palacio "neomanuelino" circundado por un vastísimo "jardín iniciático", al cual se le ha dedicado especial atención en recientes estudios iconográficos. El significado de la gramática decorativa de la Regaleira ha sido abordado desde diferentes ópticas interpretativas a fin de conocer el verdadero simbolismo del complejo. A grandes rasgos, las teorías imperantes que sustentan su significación pueden englobarse en tres apartados: las tesis mantenidas por José Manuel Anes (1991: 4-15; 1998; 2007; 2010: 99-129) y João Cruz Alves (2006) alusivas al ámbito esotérico, masónico y literario, la lectura alquímica que Rui Martins realiza en su libro A Mensagem Alquímica da Quinta da Regaleira (2013), y la reciente incursión culturalista y europeísta de la nueva disertación de Iván Moure Pazos bajo rúbrica de "El programa iconográfico de Manini y Monteiro para los jardines de la Quinta da Regaleira" (2015). Sin embargo, el empeño en la consecución de un corpus iconográfico coherente, ha "entorpecido" el análisis formal de la obra ornamental y decorativa de Manini para la Regaleira. Prueba de ello es 
que, frente a esta profusa literatura emblemática, sólo contamos con tres aportaciones formales a la gramática decorativa del complejo. Se trata de los siguientes capítulos de libros: "Traços de luz: as alfaias litúrgicas" (Carita, 2006: 152-163), “Espaço e Representação" (Pereira; Luckhurst, 2006d: 134-151) e “Il mestiere dell'Arte. Luigi Manini e la reinterpretazione del passato tra architettura e arti decorative" (Cordera, 2007: 108-115).

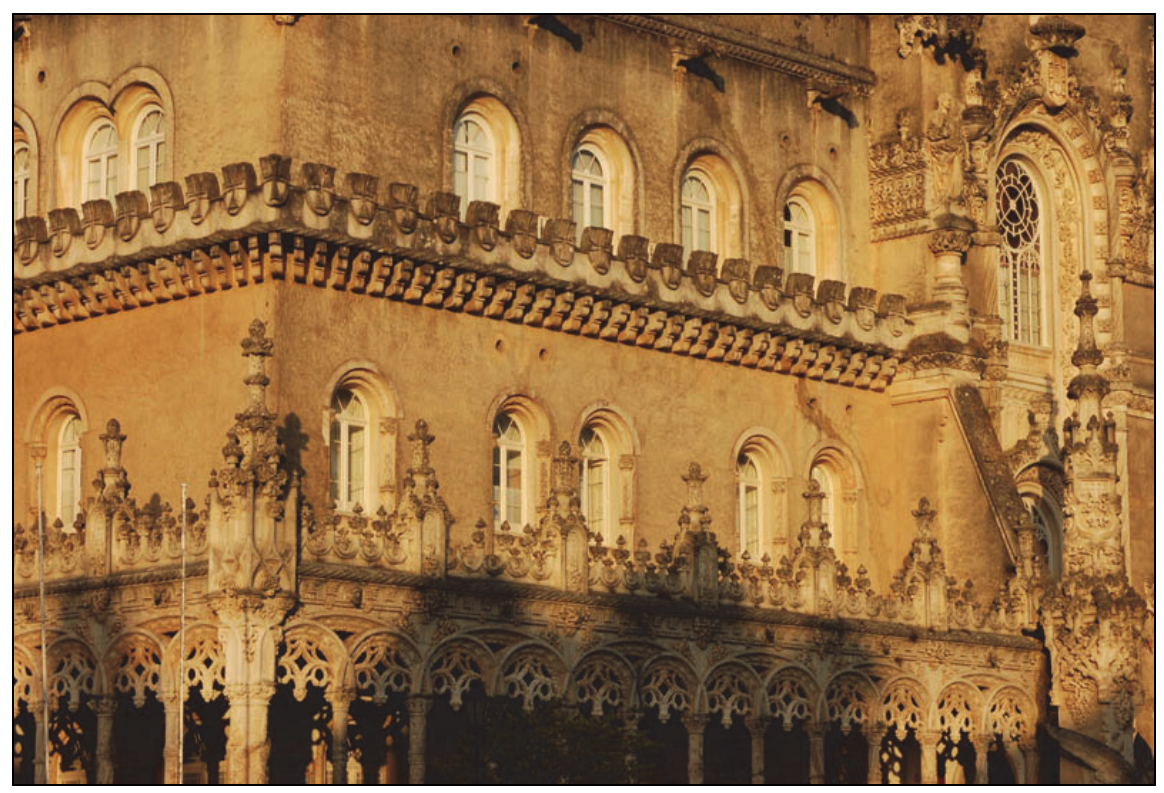

Fig. 1. Detalle del Palace Hotel de Buçaco. Foto: Javier Elcuaz del Arco

A la consabida versatilidad maniniana debemos sumarle el abordaje exitoso del léxico manuelino integrado en la Regaleira. Una vez más, Manini estudia de forma autodidacta los grandes monumentos lusos erigidos durante el reinado de D. Manuel I de Portugal (1495-1521), principalmente, La Torre de Belém (1514) y el Mosteiro dos Jerónimos (1514-1544), ambos ubicados en la ciudad lisboeta.

En el Museo Civico di Crema e del Cremasco de Lombardía se conservan decenas de diseños de molduras neomanuelinas firmadas por Manini para la Regaleira. La precisión de los trazos y el dominio estereométrico resultan sorprendentes, máxime teniendo en consideración su origen italiano. Origen, cabe apuntarlo, completamente ajeno a la nutrida cultura manuelina desarrollada en Portugal y sus territorios de ultramar en el siglo XVI. Se trata de parte del oropel ornamental con que Manini engola el suntuoso palacio sintrense, tanto en 
su revestimiento exterior como interior. Ahora bien, y como veremos en epígrafes sucesivos, Manini entrelaza este viejo lenguaje "neomedieval" de impronta portuguesa, con innúmeras concesiones al clasicismo más ortodoxo; tema que constituye el trasunto fundamental del presente artículo. Debe apuntarse que el estilo manuelino nace en Portugal en el seno de un pensamiento todavía gótico y medieval. La discreta y paulatina introducción de elementos clásicos de carácter decorativo en sus formas llevó al gran especialista Fernando Pereira Bastos a considerar dicha corriente artística como un estilo plateresco nacional (1990: 10). Sin embargo, consideramos que la concesión a lo clásico en la producción manuelina es somera en comparación con la fortísima deuda gótica que vertebra gran parte de su gramática decorativa y formal. Por lo tanto, al hablar de un manuelino ortodoxo, debemos pensar más en un "gótico con infiltraciones renacentistas" (Pereira, 1990: 11) que en un protorrenacimiento de filiaciones italianizantes y modernas. Ahora bien, Manini en la Quinta da Regaleira y quizás por su origen lombardo, carga las tintas en la parte más clásica de su obra llegando a equilibrar, como veremos, sendos lenguajes antagónicos.

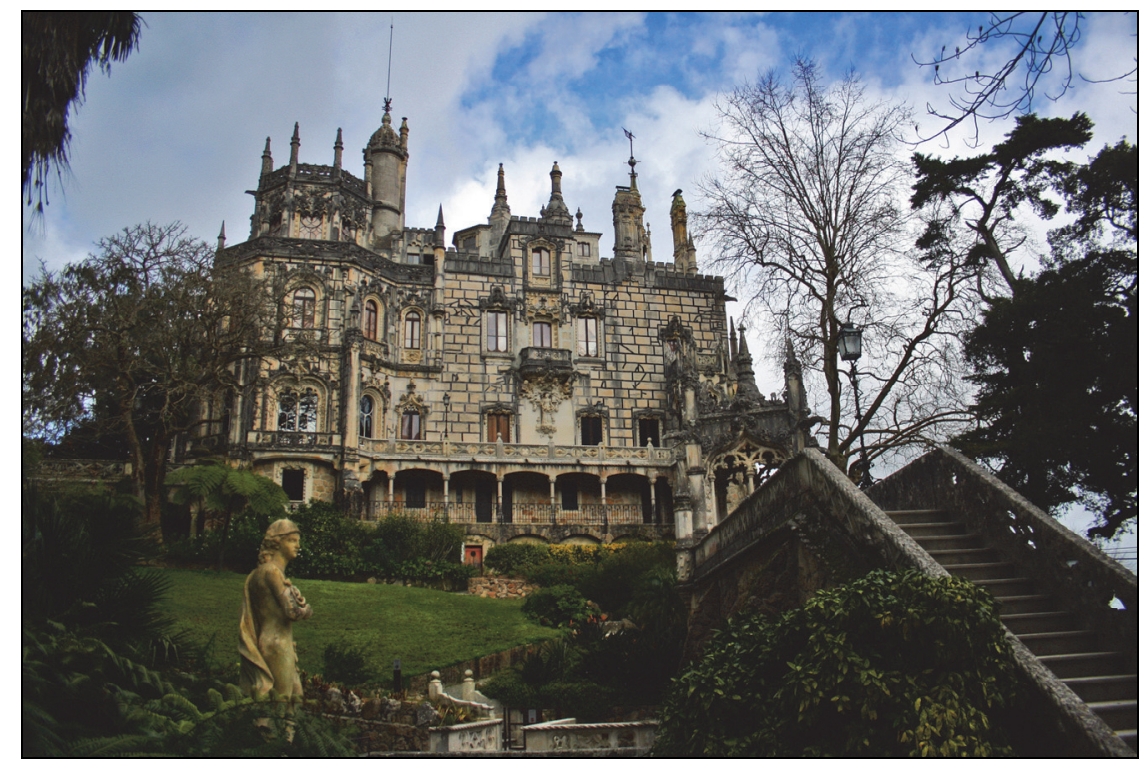

Fig. 2. Palacio principal de la Quinta da Regaleira en Sintra. Foto: Sara Gordón

Por otra parte, Manini no sólo restringe su ecléctica labor decorativa a la envoltura exterior e interior, sino que integra las artes menores y aplicadas al conjunto grupal de una "obra de arte total". Mobiliario, puertas, ventanas, 
lámparas e incluso la propia llave que daba acceso a la Regaleira fueron delineadas meticulosamente por el artista lombardo3:

Detentor de una larga experiencia como decorador que le infunde una particular sensibilidad y pericia en términos de pormenorización, Manini diseña pavimentos, techos, puertas, chimeneas, sin olvidar los detalles de los tiradores y grifos, llegando incluso a diseñar el equipamiento, mesas, sillas, armarios e incluso aperos litúrgicos para la Capilla. En esta misma línea, hasta la llave de la puerta mereció una particular atención [....] (Carita, 2006a: 122).

Prosiguiendo con la ejecución detallista y rigurosa de una obra de inmejorable factura formal, Manini encomienda sus diseños a los mejores especialistas siguiendo un patrón de excelencia pormenorizada. En Coimbra trabaja con los mejores cerrajeros, ebanistas y escultores. En Milano y Venezia con los más refinados cinceladores, vidrieros, y profesionales musivarios. António Gonçalves, João Machado, Rodrigo de Castro, Costa Mota Tio, José de Sousa Barata, António Gomes, Guiseppe Guelfi, Atelier Corvaya Bazzi e Caㅡ, y la oficina Pauly de Venezia, componen un elenco artístico difícilmente parangonable (Carita, 2006a: 117). Se trataba del principal equipo técnico, cuidadosamente seleccionado por Manini para la elaboración y materialización de sus propuestas en la Regaleira.

\section{Diseño exterior: predominancia neomedieval y contaminaciones clásicas (e hibridación de ambos léxicos).}

Durante más de 14 años, Manini dirige con empeño una vastísima y polifacética empresa artística. Se trataba de una obra de arte coral. Una enorme "jaula de hierro" actuará como esqueleto estructural del edificio, sustentando la elevadísima carga decorativa del programa ornamental, toda vez que permitirá la desmaterialización de la superficie mural a través de la sucesión de vanos "agujereando" las cuatro caras del pedernal (Pereira; Luckhurst, 2006d: 137). Se trata de

\footnotetext{
${ }^{3}$ Como curiosidad, debemos reseñar que el Mausoleo de los Carvalho Monteiro en el Cemitério dos Prazeres de Lisboa (1905-1912) -proyectado también por Manini- presenta una similitud pareja con la Regaleira: la llave que proporciona el acceso a sendos lugares es la misma. Asunto que, por supuesto, no es fruto de la casualidad, el reciclaje, o la rentabilidad artística. Antes bien y tratándose del binomio intelectual Monteiro-Manini, haremos bien en pensar en la extensión de su programa iconográfico, basado en la existencia de dos mundos interconectados: la Regaleira (morada efímera de los Monteiro durante sus vidas) y el Mausoleo (morada eterna de los mismos tras su muerte). Este detalle pudiera proporcionarnos algunas pistas sobre la planificación escrupulosa del proyecto maniniano para la Regaleira que cabe considerar de anancástico.
} 
conseguir un efecto de ligereza tectónica de filiación claramente neomedieval. Sobre este armazón gótico se superpone una gramática decorativa fácilmente reconocible. Esencialmente, se dispone gran parte del arsenal emblemático neomanuelino: heráldicas regias, cruces de la orden de Cristo, esferas armilares, redes, conchas, algas, cuerdas y nudos de cabotaje, elementos vegetales y naturales... Pero en la Regaleira todo este léxico se entrevera, en filigrana, sin orden ni concierto aparente con otras "contaminaciones iconográficas" que nada tienen que ver con el mundo medieval, trasladando al espectador a una suerte de enriquecedor paroxismo visual y barroquizante muestrario referencial. Las alusiones decorativas a la obra de Camões, Dante, Buller-Lyton, Francesco Colonna, o Fulcanelli, por no hablar de las continuas referencias esotéricas al Mesianismo, Profestismo, Templarismo, e incluso Sebastianismo, nos alejan de un neomanuelino de corte oficial u ortodoxo. Todo ello, acerca el programa decorativo de la Regaleira a las recientes tesis de Moure Pazos, sustentadas en base a hipótesis culturalistas, promovidas por los principales artífices de la obra, a los que se les presupone la creación intencionada de una gran enciclopedia en piedra de la cultura occidental, con claros fines pedagógicos e ilustrados (2015: s/p). Pero lo cierto es que, incluso desde un punto de vista meramente formal, el programa decorativo de la Regaleira debe encuadrarse dentro de la designación de un neomanuelino heterodoxo, ecléctico o si se prefiere "exótico".

Llegados a este punto debemos incidir en algo sobre lo que pocos especialistas han reparado y que consideramos sumamente importante destacar (Pereira; Luckhurst, 2006d: 138). Manini y su equipo artístico, haciendo gala de una nutrida y envidiable tradición figurativa italiana, introduce formas paganas y clásicas en un contexto predominantemente neomanuelino. Esto se evidencia en las llamadas Gruta de Leda y el Cisne y la Terraza de los Dioses. Ambas se sitúan en los vastos jardines del recinto y son continuo motivo de estudio iconográfico por parte de numerosos especialistas. Pero este lenguaje clásico continúa, tímidamente, invadiendo la decoración externa del Palacio, como podemos ver en la portada que da acceso al vestíbulo principal [Fig. 3]. Salpicado por bóvedas de crucería, delits góticos, cruces, cuerdas y todo tipo de rocaille de factura medievalizante, emerge un enmarque biselado de gruteschi a candelieri con tondos centrales, follajes, guirnaldas, mascarones y vasijas en relieve al más puro estilo renacentista. Se trata de una "contaminación clásica" muy bien integrada en el ornamento medieval, inspirada en sus primeros estudios de modelaje en la Accademia di Belle Arti di Brera. 


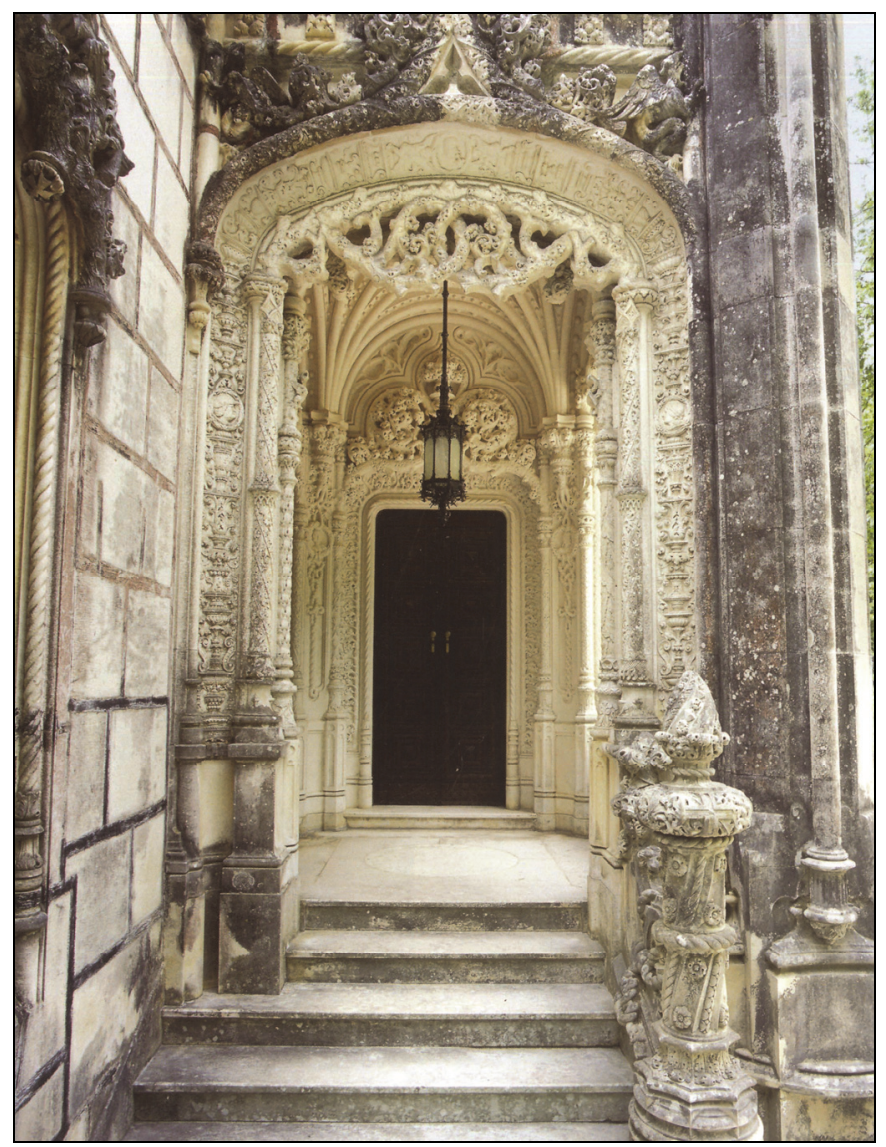

Fig. 3. Portada que da acceso al vestíbulo principal de la Quinta da Regaleira en Sintra. Foto anónima. Fuente: Catálogo exposición: Luigi Manini (1848-1936) architetto e scenografo pittore e fotografo, Piccarolo Gaia e Ricci Giuliana (coord). Milano: Silvana Editoriale. (Crema, Citadellla della Cultura, 6 maggio-8 luglio, 2007), p. 199.

Manini, como veremos, pese a los innegables logros obtenidos en terrenos poco explorados del diseño y el ornamento italiano, nunca logró desprenderse de su pasado clásico. Concretamente, su obra fue siempre deudora de los manuales que, a modo de plantillas ornamentales, se utilizaban en dicha academia durante su curso de formación. Nos referimos a los tratados de Giocondo Albertolli (1782; 1787; 1796; 1805), Domenico Moglia (1837) y Ferdinando Cassina (1840). De hecho, Giulina Ricci ha localizado la obra temprana de Manini que referencia, de manera explícita, esta nutrida tratadística (2007b: 17). Se trata de producciones imitativas de poca originalidad y factura totalmente clásica: vasos, acróteras, acantos.... Para Manini, dichos manuales supusieron la base de su formación ornamental, del mismo modo que las obras de Viollet-le-Duc, (1868; 1874), Archimede Sacchi (1874) o Camilo Boito (1880) configuraron su autodidacta cultura arquitectónica. El enmarque clásico del acceso al vestíbulo de la 
Regaleira ofrece, por lo tanto, claras concomitancias formales con los diseños de Albertolli, Moglia y Cassina. De la misma manera que el balcón central del alzado sudeste, -que luce la escultura de la nieta de Carvalho Monteiro- pudo inspirarse en los púlpitos de Domenico Moglia para su célebre Collezione di soggetti ornamentali ed architettonici inventati e disegnati da Domenico Moglia (1837: 40) en su compleja readaptación neomanuelina [Fig. 2]. Esto no es baladí, pues nos encontramos ante una de las características principales de la obra maniniana para la Regaleira: la configuración de un nuevo lenguaje ornamental, reinventado en base a la mezcla de formas clásicas y medievales. Ejemplos palmarios de esta hibridación son los innúmeros mascarones que decoran el complejo. Se trata de figuras bien reconocibles -retratadas hasta la saciedad en la obra de Albertolli (il vecchio)-, de cuyas bocas brotan, en exquisita filigrana, diversos motivos vegetales de orden clásico. Sólo que ahora, Manini sustituye los acantos de dichas bocas por una amalgama de algas manuelinas, subvirtiendo así el ortodoxo patrón figurativo grecorromano exhibido por Albertolli. Por lo tanto, Manini, al modo de los grandes diseñadores manuelinos del XVI, no sólo introduce formas clásicas en un contexto medieval, sino que hibrida ambos léxicos a fin de armonizar el conjunto ornamental. Se trata del punto de fusión, de la bisagra estilística entre dos tradiciones figurativas contrapuestas. El exterior de la Regaleira, pese a ofrecer una gran variedad de lenguajes ornamentales, totaliza en un conjunto unitario de predominancia mayormente medieval. Esto se debe, precisamente, a la premeditada y magistral gradación estilística desarrollada por el artista cremonense, conseguida mediante estas integradoras hibridaciones figurativas.

\section{Diseño interior: predominancia clásica y contaminaciones neomedievales.}

La maestría de Manini a la hora de entreverar lenguaje clásico y medieval alcanza su mayor grado de interacción artística en los interiores de la Regalei$r a$. En el vestíbulo, Manini reproduce las crucerías del portal Sur del Mosteiro dos Jerónimos (Pereira; Luckhurst, 2006c: 138) trufado con motivos manuelinos, esculturas clásicas y, nuevamente, gruteschi a candelieri. Con especial astucia, Paola Cordera ha puesto en relación el Palazzo Bagatti Valsecchi de Milano (1883) con algunos elementos decorativos diseñados por Manini para Carvalho Monteiro en la Regaleira (2007: 109). La autora establece ciertas concomitancias artísticas entre la obra cumbre del neomanuelino luso y la producción más representativa del neorrenacimiento italiano: “Emblemática, por ejemplo, 
la correspondencia entre el motivo decorativo de las cabezas de león en la puerta de ingreso al palacio Milanés y el batiente maniniano para la puerta de la Sala dos Reis en la Regaleira" (Cordera, 2007: 109). Se trata de un motivo de matriz raffaellesca de larga tradición artística italiana que Manini, a no dudar, conocía muy bien ${ }^{4}$. Otra prueba incuestionable que no debiéramos pasar por alto, es que estos prótomos figuraban en varias de las láminas del citado manual de Domenico Moglia (1837: 3, 7, 11, 17, 22, 27), manual que, cabe recordar, contribuyó sustancialmente al desarrollo formativo de Manini en Brera.

Incidiendo en esta misma línea argumentativa, Denise Pereira y Gerald Luckhurst ahondan en la correspondencia formal entre la Sala dos Reis de la Regaleira y algunas de las estancias del Palazzo Bagatti Valsecchi de Milano:

Para este espacio, Manini se inspiró en un tipo de decoración de raíz típicamente italiana. En el techo se representan sobre un fondo de hojas de oro, veinte reyes y dos reinas de varios linajes de la monarquía portuguesa: Borgonha, Aviz y Bragança. Este tipo de decoración de techo, responde a la designación de tavoleti rinascimentali, siendo una decoración típica de Lombardía. Las pinturas de ajedrezado vegetal y el primer Brasão (escudo de armas) que reposaba sobre la bellísima chimenea neorrenacentista, en eje a la sala, están inspiradas en el Quattrocento veneziano y en la réplica del XIX del palacio milanés Bagatti Valsecchi (2006c: 151).

Si bien secundamos las tesis de estos grandes especialistas, resulta difícil, como veremos, concretar las fuentes originarias en las que se inspira el artista cremonese. El Palazzo Bagatti Valsecchi se inaugura en 1883, cuatro años después de que Manini "desembarque" en Portugal. No tenemos constancia explícita de que el artista visitara dicho complejo, sin embargo, sabemos por Paola Cordera que Manini conocía dicho Palazzo a través de revistas especializadas, publicadas previamente a su encargo en la Regaleira (2007: 109). Es factible que Manini viese en este magnífico pedernal neorrenacentista la perfecta materialización de sus enseñanzas clásicas de Brera. Y es que el Bagatti Valsecchi no es más que el culmen de un neorrenacimiento que "estalla" a mediados de siglo en Italia -con especial énfasis en la región lombarda- con una larga lista de precedentes artísticos a sus espaldas. Por lo tanto, gran parte de

\footnotetext{
${ }^{4}$ Véase como ejemplo ilustrativo la cabeza de león en la chimenea de la Sala del baile de su proyecto para Buçaco (1890).
} 
los motivos decorativos y ornamentales vistos en el palacio milanés pueden rastrearse en aportaciones preexistentes ya que, como bien apunta Roberto Cassanelli (1998: 230) "los años 50 del siglo XIX señalan, de hecho, para la Lombardía, el comienzo de la difusión del gusto neorrenacentista de ornamento bramantesco y la revalorización decisiva de las lecciones de los primitivos -refiriéndose a Leonardo y su escuela-". Y más específicamente señala a la escuela de Brera como centro neurálgico de esta renovación durante los años de formación de Manini (Cassanelli, 1998: 234). Por ello no ha de extrañarnos que el entorno artístico de los hermanos Fausto e Giuseppe Bagatti Valsecchi y Luigi Manini bebieran de las mismas fuentes locales para sus respectivos proyectos, siendo en ocasiones difícil discernir entre inspiración, traducción, tradición y copia.

La misma problemática presenta la llamada Sala da Renascença en la Regaleira que, como su propio nombre indica, se dedicada por entero al Quattrocento y Cinquecento italiano [Fig.4]. Como es lógico, en dicha sala se explicita, más si cabe, el lenguaje clásico a través del icónico recetario renacentista: policromías de mármoles, affreschi, gruteschi, y una enorme chimenea renacentista que Pereira y Luckhurst (2006c: 143) han puesto en relación con su correspondiente artístico del Palazzo Ducale di Urbino (1444). Tesis secundada, nuevamente, por la profesora Paola Cordera (2007: 109).

Así, pues, como veremos a continuación, las obras en las que se inspira Manini para la recreación de los interiores de la Regaleira, constituyen un auténtico estado de la cuestión sobre la rica pervivencia de la tradición figurativa italiana. Por ejemplo, el magnífico artesonado de casetones octogonales que Manini diseña para la Sala da Renascença y que vemos nuevamente reproducido en la Camera del Letto Valtellinese del Palazzo Bagatti Valsecchi (1883), encuentran su origen en el diseño de las cornisas del Palazzo Belgioioso en Milano (1772-1787) recogidas por Giacomo Albertolli en su Alcune decorazioni di nobili sale ed altri ornamenti di Giocondo Albertolli: Professore nella Reale Accademia delle Belle Arti in Milano. Incisi da Giacomo Mercoli e da Andrea de Barnardis (1728: s/p); manual de cabecera, como sabemos, de Manini en Brera. Pero superando todo revival, debemos reseñar que la fuente originaria de este característico artesonado octogonal visto en la Regaleira forma parte firmante y fundamental de una de las obras más icónicas del Quattrocento florentino. Nos referimos a los cassettoni de la Sala dell'Udienza (1470-1476) y la Sala dei Gigli 
(1470-1472) realizados por los hermanos Benedetto y Giuliano da Maiano para el Palazzo Vecchio de Firenze [Figs. 4-8]

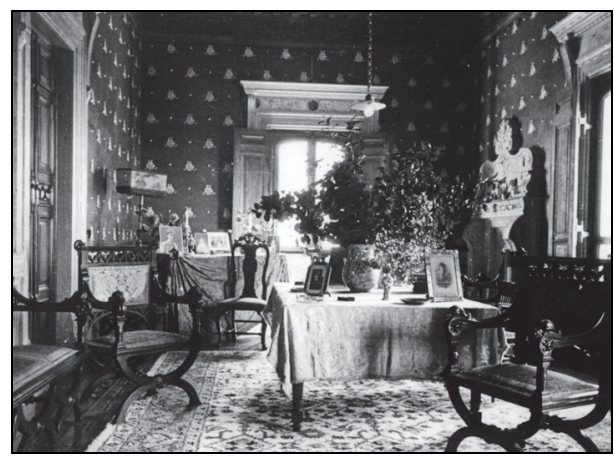

Fig. 4. Sala da Renascença de la Quinta da Regaleira en Sintra. Foto de época (1945). Fuente: Fundação Cultursintra.

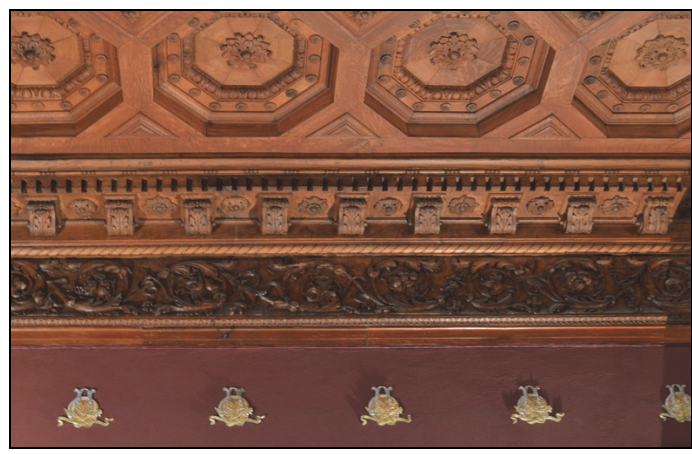

Fig. 5. Detalle del artesonado de la Sala da Renascença de la Quinta da Regaleira en Sintra. Fuente: O que vi do mundo.

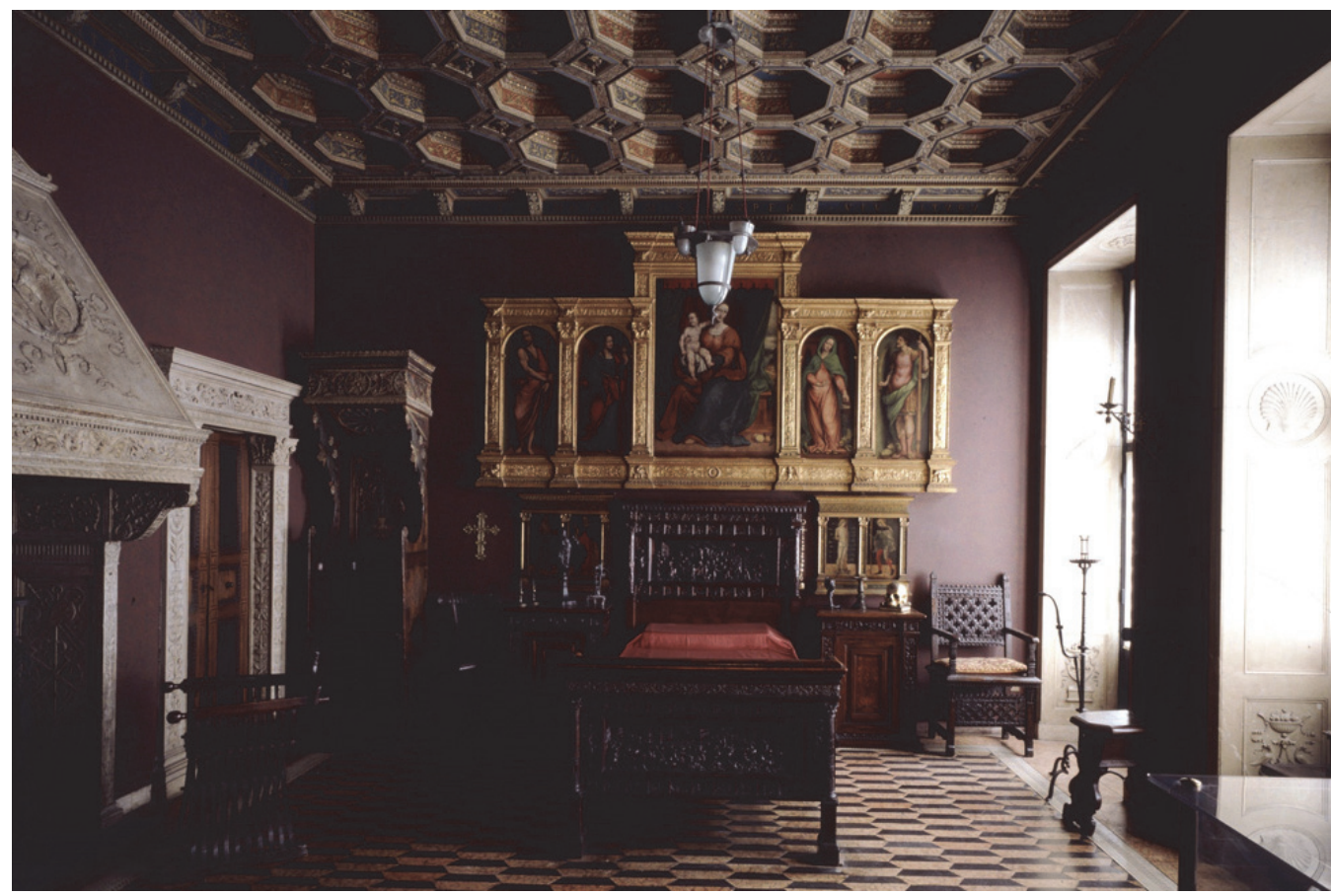

Fig. 6. Camera del Letto Valtellinese del Palazzo Bagatti Valsecchi en Milano. Fuente: Museo Bagatti Valsecchi 


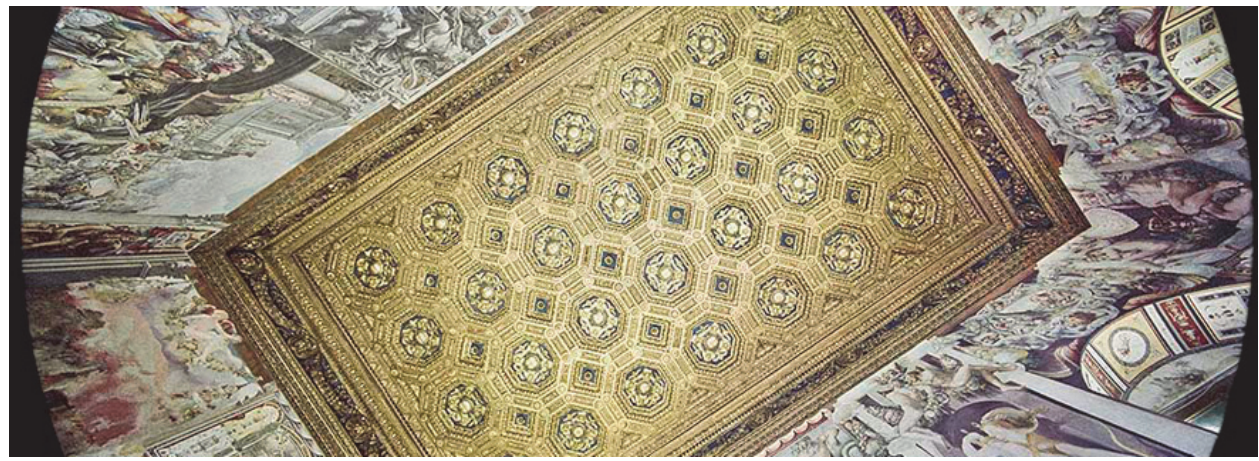

Fig. 7. Detalle del artesonado de la Sala dell'Udienza del Palazzo Vecchio en Firenze. Foto: Franco Zampetti

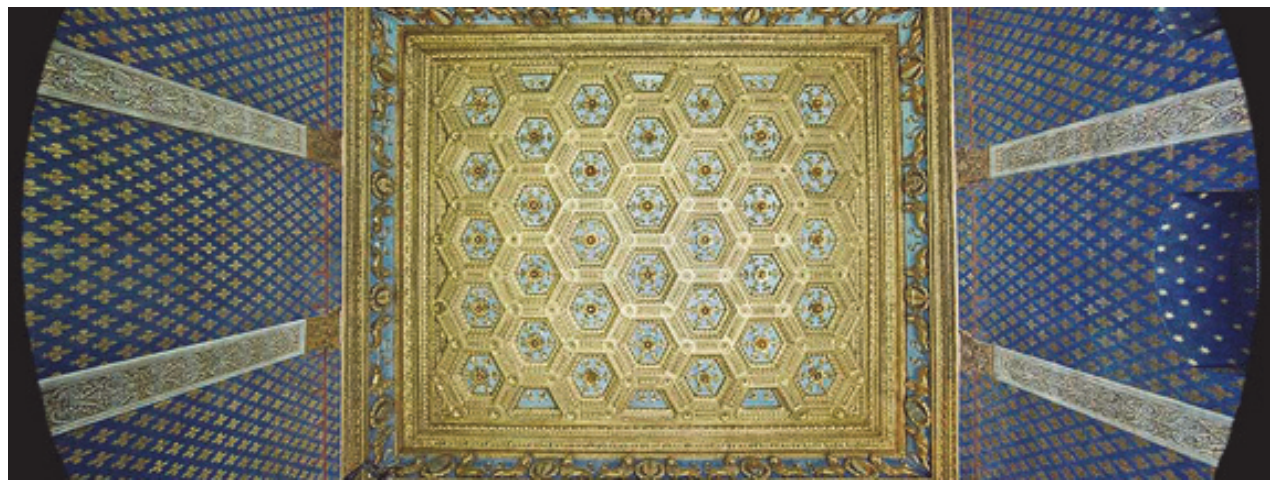

Fig. 8. Detalle del artesoando de la Sala dei Gigli delPalazzo del Palazzo Vecchio en Firenze. Foto: Franco Zampetti.

Asimismo, los vanos diseñados por Manini para la Sala da Renascença, pudieran inspirarse, nuevamente, en la tratadística albertolliana, referida a las láminas tituladas Spaccato per il lungo della sala de' pranzi della Real Villa di Monza, Ornamento di porta eseguito in legno per una delle sale della Villa Reale di Monza, y Ornamento di porta, e ossatura dello specchio con tavolino, che viene dirimpetto alla camminiera; cornice di tappezzeria, e basamento, il tutto eseguito in legno nella sala accennata nel foglio precedente (1728: s/p). Del mismo modo que la célebre chimenea, pudiera parangonarse tanto al Camino eseguito in marmo di Carrara per una delle sale della R. Corte in Milano (Albertolli, 1728: s/p), como a las célebres camini mostrados por Domenico Moglia en su tradística (1837: 34, 35). Sea como fuere, debemos añadir al citado símil de la chimenea de la Sala de Ercole e Jole del Palazzo Ducale di Urbino de Michele di Giovanni da Fiesole 
(Il Greco) -puesto en solfa por tres grandes especialistas en Manini (Pereira; Luckhurst, 2006c: 143; Cordera, 2007: 109)- las siguientes referencias fundamentales: la chimenea del Palazzo Gondi en Firenze (1490) realizada por Giuliano Sangallo y la subsecuente del Palazzo Borgherini-Rosselli del Turco en la misma ciudad (1507) firmada por Benedetto da Rovezzano [Figs. 9-12].

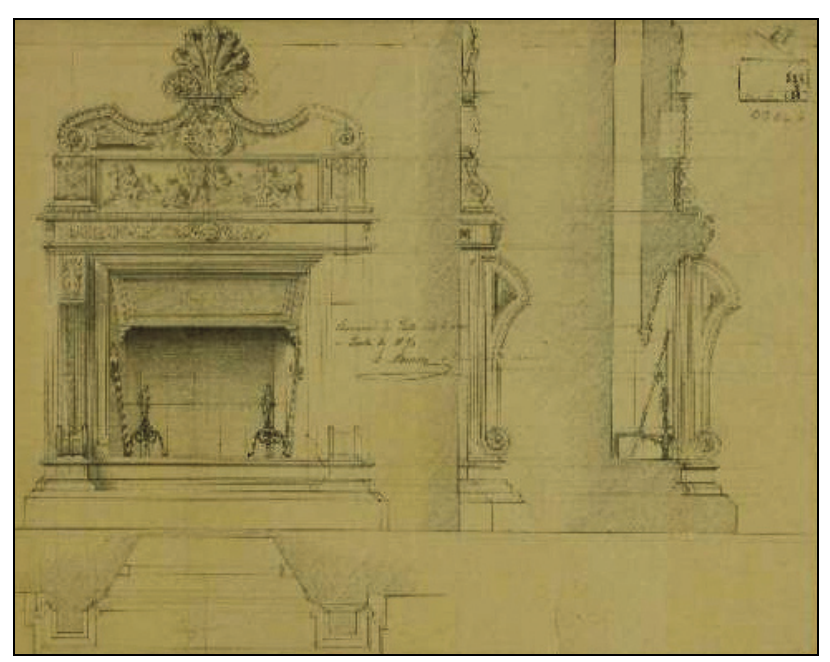

Fig. 9. Alzado de la chimenea de la Sala de la Renascença de la Quinta da Regaleira en Sintra. Museo Civico di Crema e del Cremasco.

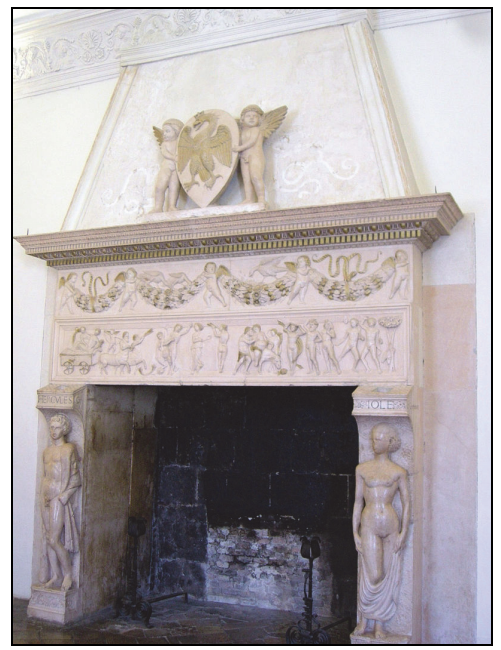

Figs. 10. Chimenea de la Sala de Ercole e Jole del Palazzo Ducale di Urbino. Foto: Sailko

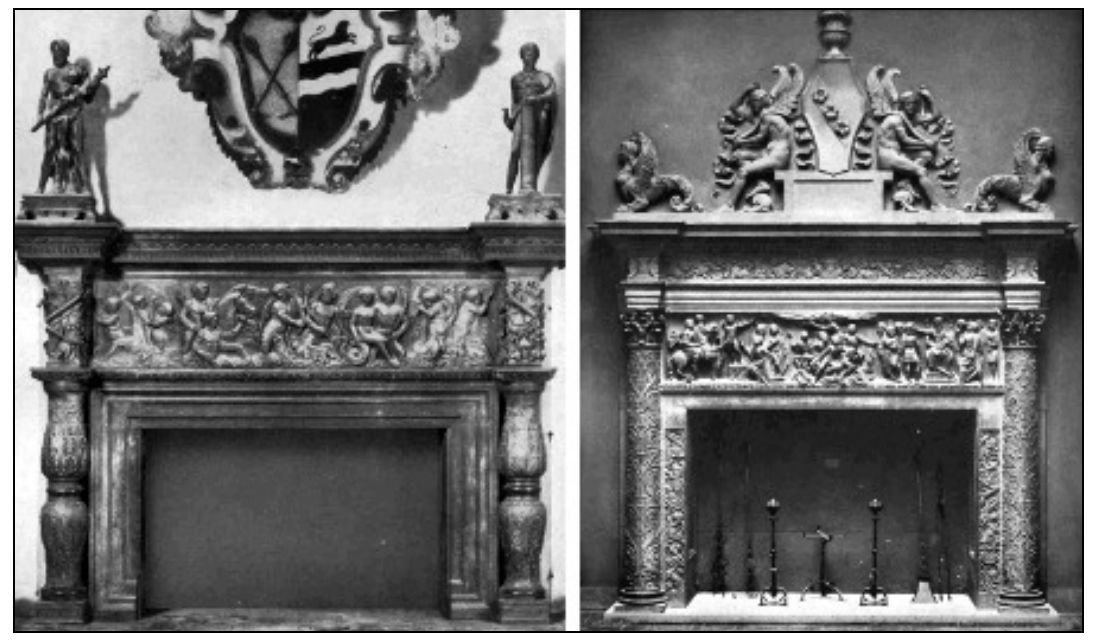

Figs. 11-12. Chimeneas de los Palazzi Gondi y Borgherini-Rosselli del Turco en Firenze. Foto de época. Fuente: Fondazione Federico Zeri. 


\section{Conclusiones.}

Después de todas estas páginas, resulta evidente que gran parte de la retórica ornamental manejada por Manini en la que es considerada como la obra más representativa del neomanuelino portugués -amén de su gran aportación a Buçaco-, se articula en torno a una gran variedad de elementos clásicos que dirimen más en una hibridación ecléctica muy bien equilibrada de pensamiento medieval y clásico que en un neomanuelino ortodoxo de clara predominancia gótica. Esto se hace más evidente en los interiores del pedernal. Buen, ejemplo de ello es que de las más de 15 estancias que componen la Regaleira en su piso noble, 11 poseen pavimentos y techumbres de orden clásico.

El Manuelino, estilo de grandes edificios públicos y religiosos, pudiera resultar un tanto excesivo en su ornamento para la decoración interior de una Quinta de uso privado; incluso para una Quinta de estas dimensiones. Sin embargo, la sobriedad, el equilibrio y la proporción decorativa de las grandes viviendas burguesas italianas ofrecieron a los Monteiro un mejor prototipo de habitabilidad y mesura habitacional para el desarrollo de sus quehaceres cotidianos. La solución maniniana no podría estar mejor estructurada: el exterior, se erige como un imponente palacio "medieval" irrumpiendo en la sierra sintrense como un gran castillo encantado, con sus gárgolas, pináculos y torres alquímicas; el interior, ofrece al estudioso una experiencia menos lúgubre, más equilibrada, de corte bramantiana, sosegante y pacificante.

Manini ha pasado a los anales de la historia del arte por ser el artista más representativo del neomanuelino luso, convirtiéndose en figura icónica del revival medieval en las postrimerías del siglo XIX. Sin embargo, y como hemos demostrado a lo largo de este artículo, su lenguaje italianizante y clásico aflora con fuerza en su faceta de excelente diseñador de ornamentos; incluso en aquellos proyectos exaltadores del ánima lusitana neomanuelina que requerían de una mayor concesión hacia lo gótico. Con motivo del octogésimo aniversario de su fallecimiento, creemos interesante recordar a Manini por su aportación artística menos celebrada: aquella que tiene que ver con las reiteradas y sucesivas concesiones al lenguaje ornamental clásico y su genial confluencia con el mundo "medieval" portugués. Maravillosa hi- 
bridación estilística de la que la Regaleira -actualmente Patrimonio Mundial de la Humanidad UNESCO- hace gala gracias a la capacidad innovadora y creadora del genio cremonese.

\section{Referencias bibliográficas.}

Albertolli, G. (1782): Ornamenti diversi, inventati disegnati ed eseguiti da Giocondo Albertolli: Professore d'Ornati nella Reale Accademia di Belle Arti in Milano. Incisi da Giacomo Mercoli Luganese. Milano: si vendono dallo stesso Albertolli.

Albertolli, G. (1787): Alcune decorazioni di nobili sale ed altri ornamenti di Giocondo Albertolli: Professore nella Reale Accademia delle Belle Arti in Milano. Incisi da Giacomo Mercoli e da Andrea de Barnardis. Milano: S.E.

AlBertolli, G. (1796): Miscellanea per i giovani studiosi del disegno pubblicata da Giocondo Albertolli: Professore nella Reale Accademia delle Belle Arti in Milano. Milano: si vendono dallo stesso Albertolli.

Albertolli, G. (1805): Corso elementare di ornamenti architettonici ad uso déprincipianti. Milano: si vendono dallo stesso Albertolli.

AlPINI, Cesare (2007): “Manini Pittore”, en PICCAROLO Gaia e RICCI Giuliana (coord), Catálogo de la exposición: Luigi Manini (1848-1936) architetto e scenografo pittore e fotografo (117-129). Milano: SilvanaEditoriale. (Crema, Citadellla della Cultura, 6 maggio-8 luglio, 2007).

ANES, J. M. (1991): “Digressão hermética por uma mansão filosofal portuguesa. in Quinto império", Jornal mensal de temática hermética, (3), 4-15.

ANES, J. M; PEREIRA, P; PEREIRA, D. (1998): A Quinta da Regaleira, história, símbolo e mito. Sintra: Fundação Cultursintra.

ANES, J. M. (2007): Os jardins iniciáticos da Quinta da Regaleira. Lisboa: Ésquilo.

ANES, J. M. (2010): “O espaço sagrado e os jardins iniciáticos da Quinta da Regaleira", Revista Lusófona de Arquitectura e Educação, (3), 99-129.

BoITO, C. (1880): Architettura del Medio Evo in Italia, con una introduzione sullo stile futuro dell'architettura italiana. Milano: Hoepli.

CARITA, H. (2006a): "A Quinta da Regaleira: Projecto e Método", en Joao CRUZ ALVES (coord), Catálogo de la exposición: Luigi Manini: Imaginário y Método, Arquitectura y Cenografia (90-98). Sintra: CulturSintra. (Sintra, 29 de Junho-31 de outubro, 2006). 
CARITA, H. (2006b): “Traços de luz: as alfaias litúrgicas”, en Joao CRUZ AlveS (coord), Catálogo de la exposición: Luigi Manini: Imaginário y Método, Arquitectura y Cenografia (152-163). Sintra: CulturSintra. (Sintra, 29 de Junho31 de outubro, 2006).

CASSANELLI, R. (1998): "Fotografia, storiografia artistica, restauro. Qualche indizio di percorsi incrociati a Milano intorno al 1850", en VV.AA, Fare storia dell'arte. Studi offerti a Liana Castelfranchi (227-236). Milano: Jaca Book.

CASSINA, F. (1840): Le fabbriche più cospicue di Milano, pubblicate per cura di Ferdinando Cassina. Milano: Cassina and Pedrinelli.

CORDERA, P. (2007): “Il mestiere dell'Arte. Luigi Manini e la reinterpretazione del passato tra architettura e arti decorative", en PICCAROLO Gaia e RICCI Giuliana (coord), Catálogo de la exposición: Luigi Manini (1848-1936) architetto e scenografo pittore e fotografo (108-115). Milano: SilvanaEditoriale. (Crema, Citadellla della Cultura, 6 maggio-8 luglio, 2007).

CRUZ Alves, J. (2006): “Iconografia e Imaginario", en Joao CRUZ AlveS (coord), Cátalogo de la exposición: Luigi Manini: Imaginário y Método, Arquitectura y Cenografia (188-222). Sintra: CulturSintra. (Sintra, 29 de Junho31 de outubro, 2006).

D’ACIE (Pseudónimo de Augusto CAMBIÈ) (1936): Luigi Manini scenografoarchitetto. Crema: tipografía la Moderna.

D’AMIA, G. (2007): “Luigi Manini scenografo. Dalla formazione scaligera ai trionfi portoghesi", en PICCAROLO Gaia e RICCI Giuliana (coord), Catálogo de la exposición: Luigi Manini (1848-1936) architetto e scenografo pittore e fotografo (53-65). Milano: SilvanaEditoriale. (Crema, Citadellla della Cultura, 6 maggio-8 luglio, 2007).

HENRIQUES DA SILVA, R. (2004): O neomanuelino do palace-hotel: pistas para pensar a memória". Monumentos, (20), 44-49.

LUCKHURST, G; PEREIRA, D. (2007): “Dalla poetica della natura alla costruzione del paesaggio e del giardino. L'interpretazione di Luigi Manini", en Gaia PICCAROLO e Giuliana RICCI (eds.), Catálogo exposición: Luigi Manini (18481936) architetto e scenografo pittore e fotografo (87-99). Milano: SilvanaEditoriale. (Crema, Citadellla della Cultura, 6 maggio-8 luglio, 2007).

MogLIA, D. (1837): Collezione di soggetti ornamentali ed architettonici inventati e disegnati da Domenico Moglia. Milano: Tip. Dottore G. Ferrario. 
Moure PAZOS, I. (2014): “Las Villas de Luigi Manini en Sintra (1890-1912)”, Ángulo Recto, Vol.6, (2), 101-116.

MOURE PAZOS, I. (2015): “El programa iconográfico de Manini y Monteiro para los jardines de la Quinta da Regaleira", (En prensa).

Pereira BASTOS, F. (1990): Apontamentos sobre o Manuelino no distrito de Lisboa. Lisboa. Lisboa: Impresa-Nacional-Casa da Moeda.

PereirA, D; LuCKHURST, G. (2004a): “Luigi Manini no Buçaco. Os passos da cenografia à arquitectura e as peripécias do processo administrativo". Monumentos, (20), 50-63.

PEREIRA, D; LUCKHURST, G. (2004b): “A Vila Sassetti, projecto do cenógrafoarquitecto Luigi Manini”, Varia Escrita, (11), 253-280.

PEREIRA, D; LUCKHURST, G. (2006a): “Luigi Manini: esboço de uma biografia”, en Joao CRUZ ALVES (coord), Cátalogo de la exposición: Luigi Manini: Imaginário y Método, Arquitectura y Cenografia (11-19). Sintra: CulturSintra. (Sintra, 29 de Junho-31 de outubro, 2006).

PereirA, D; LUCKHURST, G. (2006b): “Cenografia e espectáculo”, en Joao CRUZ ALVES (coord), Cátalogo de la exposición: Luigi Manini: Imaginário y Método, Arquitectura y Cenografia (21-63). Sintra: CulturSintra. (Sintra, 29 de Junho-31 de outubro, 2006).

PereirA, D; LuCKHurst, G. (2006c): "Entre Sintra e Cascais”, en Joao CruZ ALVES (coord), Cátalogo de la exposición: Luigi Manini: Imaginário y Método, Arquitectura y Cenografia (77-89). Sintra: CulturSintra. (Sintra, 29 de Junho-31 de outubro, 2006).

PEREIRA, D; LUCKHURST, G. (2006d): “Espaço e Representação”, en Joao CRUZ ALVES (coord), Cátalogo de la exposición: Luigi Manini: Imaginário y Método, Arquitectura y Cenografia (134-151). Sintra: CulturSintra. (Sintra, 29 de Junho-31 de outubro, 2006).

PereirA, D; LucKhurst, G. (2008). “Luigi Manini e o projecto da Vila Sassetti", Monumentos, (26), 200-210.

PEREIRA, D; LUCKHURST, G. (2011). “O programa estético da casa de Jorge O'Neill, a partir dos contributos de Luigi Manini, Francisco Vilaça e Albrecht Haupt", Monumentos, (31), 92-105.

PEREIRA, D. (2012). “A cenografia oitocentista como fonte de cultura visual e de ideologia", História da arte, (10), 147-159. 
PICCAROLO, G. (2005): Luigi Manini (1848-1936). L'opera architettonica di uno scenografo italiano in Portogallo. Tesis de Licenciatura dirigida por Giuliana Ricci, Politécnico de Milano.

Piccarolo, G. (2006): “Luigi Manini architetto. Progetti di uno scenografo cremasco in Portogallo tra XIX e XX secolo". Insula Fulcheria, (35), 389-412.

Piccarolo, Gaia (2007): “Villa e Villino: il tema dell'abitazione borghese nell'opera di Luigi Manini", en PICCAROLO Gaia e RICCI Giuliana (coord), Catálogo de la exposición: Luigi Manini (1848-1936) architetto e scenografo pittore e fotografo (99-107). Milano: SilvanaEditoriale. (Crema, Citadellla della Cultura, 6 maggio-8 luglio, 2007).

ReginA, A. (2004): "Palace-Hotel: projectos de construçao". Monumentos, (20), 64-73.

RICCI, G. (2007a): “Introduzione" en PICCAROLO Gaia e RICCI Giuliana (coord), Catálogo de la exposición: Luigi Manini (1848-1936) architetto e scenografo pittore e fotografo (14-15). Milano: SilvanaEditoriale. (Crema, Citadellla della Cultura, 6 maggio-8 luglio, 2007).

RICCI, G. (2007b): “L'archittetura come arte applicata per il benessere del popolo e della nazione" en PICCAROLO Gaia e RICCI Giuliana (coord), Catálogo de la exposición: Luigi Manini (1848-1936) architetto e scenografo pittore e fotografo (17-27). Milano: SilvanaEditoriale. (Crema, Citadellla della Cultura, 6 maggio-8 luglio, 2007).

SACCHI, A. (1874): Le abitazioni: alberghi, case operaie, fabbriche rurali, case civili, palazzi e ville: ricordi compendiati da Archimede Sacchi. Milano: Hoepli.

VALDEÓN, J. (2008): “El bosque sagrado de Buçaco”, Arquitectura del Paisaje, 2008, (172), 14-15.

VARelA GOMES; P. (2005): Buçaco: o deserto dos Carmelitas Descalços. Coimbra: XM.

VIOLLET-LE-DUC, E. (1868): Dictionnaire raisonné de l'architecture française du XIe au XVIe siècle (1854-1868). Paris: Édition BANCE - MOREL.

VIOLLET-LE-DuC, E. (1874): Dictionnaire raisonné di mobilier française de l'epoque carlovingienne à la Renaissance. Paris: Édition BANCE - MOREL. 